\title{
Neuromuscular Timing Activity on Ankle Musculature in Young Adults during Single and Dual-motor Tasks Accompanied with Altered Sensory Conditions
}

\author{
Martín G. Rosario PT, PhD, CSFI, ATRIC*, Carley Bowman, SPT, Aleena Jose, SPT
}

Physical Therapy Program, Texas Woman's University, Dallas Campus; Texas, United States.

\author{
Article Details \\ Article Type: Research Article \\ Received date: $19^{\text {th }}$ June, 2020 \\ Accepted date: $15^{\text {th }}$ October, 2020 \\ Published date: $19^{\text {th }}$ October, 2020
}

"Corresponding Author: Martín G. Rosario PT, PhD, CSFI, ATRIC, Physical Therapy Program, Texas Woman's University, Dallas Campus; 5500 Southwestern Medical Ave. Dallas, TX 75235-7299, United States. E-mail: mrosario1@twu.edu

Citation: Rosario, M.G., Bowman, C., \& Jose, A. (2020). Neuromuscular timing activity on ankle musculature in young adults during single and dual-motor tasks accompanied with altered sensory conditions. J Rehab Pract Res 1(2): 112. https://doi. org/10.33790/jrpr1100112

Copyright: (C2020, This is an open-access article distributed under the terms of the Creative Commons Attribution License 4.0, which permits unrestricted use, distribution, and reproduction in any medium, provided the original author and source are credited.

\begin{abstract}
s
Previous literature has extensively analyzed neuromuscular patterns in various neuromuscular populations as well as during complex balance conditions that involve a secondary task.

Purpose: This inquiry aims to identify whether challenging the sensory systems in young adults during single and dual-motor tasks can elicit changes in neuromuscular patterns at the gastrocnemius (GA) and tibialis anterior (TA).

Methods: Our study consisted of twenty-nine healthy young adults ( 3 males and 25 females) with an average age of 24.3+/-2.4. Electromyography (EMG) electrodes were utilized to collect EMG data. The variables of interest include the onset of muscle contraction, the decay of muscle contraction, peak muscle activation, and duration of muscle contraction. The distinct balance tests consisted of four single and four dual-motor tasks where participants were instructed to maintain balance on a foam surface while concomitantly altering their sensory input.
\end{abstract}

Results: Overall, no significant changes in EMG activity were demonstrated during the different tests, though some substantial alterations in EMG patterns were identified during dual-motor tasks. Fluctuations in EMG patterns during dual-motor tasks were much higher in GA than TA, with higher average onset during eyes open $(p=0.01)$, higher decay of contraction during eyes closed $(\mathrm{p}=0.01)$, and longer duration of activity during eyes open with head movements $(\mathrm{p}=0.001)$.

Conclusion: Though the invariability of EMG patterns during single tasks implies intact sensory and motor systems in young adults, the variability in EMG patterns during dual-motor tasks indicate the presence of dual-task interference, requiring additional postural mechanisms to maintain balance. Future studies should compare EMG response during dual-cognitive and dual-motor tasks and the cortical involvement during dual-motor tasks.

Keywords: Electromyography (EMG), Single Tasks, Dual-Motor Tasks, Balance Conditions

\section{Introduction}

Balance can be characterized in mechanical terms as the proficiency to conserve the center of gravity $(\mathrm{CoG})$ within the area of the base of of support (BoS). When CoG falls outside of the BoS, it can be assumed the person would become unbalanced and fall unless actions are taken to recover the CoG within the limits of their BoS. For the human body, the CoG is broader compared to the base of support $(\mathrm{BoS})$ in upright standing posture, requiring humans to utilize several postural control mechanisms via muscles to maintain balance and not fall [1].

Static and dynamic balance both include muscle recruitment varying from the proximal trunk muscles to the distal ends of body segments to execute postural control mechanisms. For this research, the lower extremity musculature will be what the findings pertain to. For standing tandem balance in the frontal plane, prior evidence found the largest recruitment in frontal plane stability comes from tibialis anterior (TA) and peroneus muscles to give quick feedback through reciprocal action. The soleus muscle's tonic nature assists in maintaining the body upright while balancing tasks [2].

During standing sway, peak neuromuscular activity correlation can be seen between the gastrocnemius and tibialis anterior, the deep compartment of leg musculature [3]. Similarly, when anterior sway occurs in static standing, the gastrocnemius peaks activation, but during posterior sway TA activity peaks [4]. This peak activation of either muscle is crucial for preventing falls in the anterior or posterior direction.

In addition to balance requiring several rhythmic muscle activations to remain stable, the visual, somatosensory, and vestibular systems must function in unison to maintain balance control. When one or more of these systems are not working synchronously with one another, postural instability is the outcome. Researchers utilize motor control theories such as the dynamic system theory to comprehend the interplay between the sensory systems. This notion illustrates how the three sensory systems need to interact efficiently to maintain dynamic or static balance, even when the sensory systems are challenged [5]. When one system or systems are challenged, or if there are impairments in one or more of the systems, sensory reweighting occurs, where the other systems will take over to maintain posture [5]. Various pathologies can bear upon the balance system from vestibular input to proprioceptive information of the surroundings. For example, diabetes mellitus (DM) can be accompanied by several comorbidities like polyneuropathy, retinopathy, vestibular dysfunction, 
and polypharmacy, all of which can compromise different components of the balance system. With each of these pathologies, individuals with DM can find it more problematic to integrate sensory information to formulate effective balancing strategies leading to a higher frequency of falls [6].

Balance can be assessed and quantified with various measurements and devices; for the purposes of this inquiry, the objective measurements will be assessed. Objectively, balance measurements can be achieved with the use of force platforms, gyroscopes, and electromyography (EMG) analysis. Force platforms can analyze and track the center of pressure $(\mathrm{CoP})$, how it becomes displaced, and convert these measurements via calculations into postural control measurements. Other measurements, such as Gyroscopes and Accelerometers are designed to capture the stance and orientation of a subject, combined with the non-gravitational velocity during dynamic and static balance [7]. Finally, EMG analysis utilizes small electrodes adhered to the surface of the skin to enable the capturing of the intensity and timing of superficial muscle contractions in static or dynamic balancing tasks [8]. Overall, when examining balance, several factors must be brought into consideration, such as time available to administer the test, funds accessible to the clinician/ researcher, and balance analytics desired for the individual.

Clinicians often incorporate dual-tasking into fall prevention treatment plans for fall risk patients. Dual tasks can be described as an individual's ability to perform two or more cognitive and motor activities simultaneously while maintaining an upright posture. For the purposes of this research, motor activity manipulation will be the focus. These are important considerations during discharge planning to ensure patients are able to return to their activities of daily living (ADLs), which oftentimes require dual-motor tasks [9]. The study by Shin \& An exhibited a significant increase in balancing abilities and reduction in postural sway, exemplifying improved balance due to dual motor tasks [9]. Another study assessed the ability of chronic stroke patients to dual motor task by having participants sit on uneven terrain while simultaneously performing upper extremity activities. The results of this study discussed the ability of dual motor task training to increase trunk muscle recruitment in 3-dimensional planes such as extension, lateral flexion, and rotation versus 1-dimensional movement planes. In conclusion, the dual-motor tasks in 3-dimensional planes demanded greater stability [10]. These two studies exemplify the positive effects dual-motor tasks can have on improved balance and increased muscle recruitment.

Previous literature has identified alterations in EMG activity and postural sway during distinct balance tests in individuals with neuromuscular disorders. Although most studies have compared balance responses during single-tasks and dual-cognitive tasks, the literature regarding balance response during dual-motor tasks is limited. Our study aims to investigate whether neuromuscular activation patterns can be altered in young healthy adults during single and dual-motor tasks in conjunction with altered sensory conditions. We speculate that EMG activity of tibialis anterior and gastrocnemius will be invariable during the single-tasks due to the simplicity of the task, but variations in EMG activity will be seen during dual-motor tasks which require more multitasking efforts.

\section{Methods}

Participants were obtained via convenient sampling at the Texas Woman's University Dallas campus, where all data collection occurred as well. Each participator signed an informed consent upon agreement after a research assistant explained the study's objectives. Then participants were screened to obtain demographic data such as weight, height, age, and foot dominance.

It was required participants to be young healthy adults within the age range of 18-45 years old to partake in the study, to avoid any agerelated variations in balance control.

The following exclusion factors were established to avoid any confounding factors that could alter EMG activity: 1) unstable postural control for 30 seconds during the Romberg Test, 2) women who are pregnant or think they are pregnant, 3) BMI $>40$ 4) individuals with hypertension, 5) any untreated visual acuity or difficulty reading small prints even while wearing prescription glasses, 6) any surgeries or injuries at the back or lower extremity within the past 6 months, 7) any use of drugs with side effects of drowsiness that endures more than 24 hours prior to our study.

Participants: Table 1 demonstrates the participants' demographic information. A total of 28 participants ( 25 females and 3 males with an average age of $24.3+/-2.4$ years) participated in this study. The dominant leg of each participant was determined for the placement of electromyography (EMG) electrodes.

\begin{tabular}{|l|l|}
\hline Characteristics & Control Participants Average $\mathrm{n}=28$ \\
\hline Age & $24.3+/$ - 2.4 years \\
\hline Gender & Male $=3$ Female $=25$ \\
\hline Height $(\mathrm{in})$ & $65.7+/-3.06$ \\
\hline Weight $(\mathrm{lb})$ & $144.9+/-25.9$ \\
\hline Heart Rate $(\mathrm{bpm})$ & $70.8+/-15.5$ \\
\hline Systolic BP $(\mathrm{mmHg})$ & $111.4+/-14.2$ \\
\hline Diastolic BP $(\mathrm{mmHg})$ & $73.2+/-6.8$ \\
\hline Sat O2 (\%) & $98.3+/-0.9$ \\
\hline Dominant Leg & $\begin{array}{l}\text { Right }=20 \\
\text { Left }=8\end{array}$ \\
\hline
\end{tabular}

Neuromuscular Assessment: For the electromyography surface electrode system (EMG) (Delsys, Inc. Boston, MA), the current study attended the position of the electrode on the leg muscles based on Sacco \& Kasman guideline. EMG was employed to obtain electrical activity of muscles at $1,000 \mathrm{~Hz}$. The electrical activity of the gastrocnemius (GA) and tibialis anterior (TA) was of interest in this investigation. A razor was utilized to eliminate any excess hair and clean the area at the electrode placement site to circumvent any any impedance to EMG data transference.

Subjects sustain a static bipedal stance while concurrently gazing at a square at eye level 10-feet away. In this study, nine tasks were designed to examine balance and the EMG modifications on leg muscles. One of the tasks was performed on a firm surface while the other eight were conducted on a foam pad (15.5 inches long, 12.5 inches wide, 2.4 inches height) placed on a firm surface. The balance 
examinations consisted of four single tasks and four dual-motor tasks (holding a $12 \mathrm{oz}$ cup filled with water). The EMG activity for each balance test was recorded for 15 seconds. In this research, we identify the onset of maximal activity, when the muscle contraction sinks or decays (Decay), peak muscle activation (TP), and duration of muscle contraction (Duration) as our variables of interest.

Balance Assessment: The single-task balance tests on foam were as follows: 1) eyes open (EO), 2) eyes closed (EC), 3) eyes open with head movements (EOHUD) with a cadence of 60 beat per minute, 4) eyes closed with head movements (ECHUD). The same balance examinations were executed during the dual-motor tasks, although participants were asked to hold a cup filled with water while concurrently executing the tests.

\section{Data Analysis}

EMG data was captured by EMG works and processed by EMG analysis for both the TA and GA musculature. For the data analysis, this study utilized SPSS (version 25) with a Wilcoxon Signed Rank Non-Parametric Test for the comparison within TA during single and dual tasks, and GA while single and dual tasks. Further, to comprehend the neuromuscular coordination and adjustment we implemented a Wilcoxon Signed Rank Non-Parametric Test to associated TA versus GA activation during single and dual tasks. Subsequently, the current investigation compares all neuromuscular-time (onset, time to peak, decay, and duration) variables across each task. To accommodate to the repeated analysis, we consider a $p$-value of $<0.01$ as statistically significant.

\section{Results}

Table 2 demonstrates no significant differences in GA activation during single and dual tasks. Table 3 also shows similarities of TA activation during different tests, though the average duration of activity during EOHUD was significantly higher during single tasks compared to dual tasks $(\mathrm{p}=0.001)$.

\begin{tabular}{|c|c|c|c|c|c|}
\hline $\mathrm{N}=29$ & $\begin{array}{l}\text { Dual Task } \\
\text { Means }\end{array}$ & $\begin{array}{l}\text { Dual Task } \\
\text { SD }\end{array}$ & $\begin{array}{l}\text { Single Task } \\
\text { Means }\end{array}$ & $\begin{array}{l}\text { Single Task } \\
\text { SD }\end{array}$ & P Value \\
\hline Onset-EO & 5.24 & 4.35 & 5.98 & 3.74 & 0.46 \\
\hline TP-EO & 1.08 & 0.86 & 1.19 & 1.31 & 0.91 \\
\hline Decay-EO & 1.40 & 1.04 & 1.36 & 1.18 & 0.63 \\
\hline Duration EO & 2.48 & 1.59 & 2.55 & 1.70 & 0.86 \\
\hline Onset EC & 7.97 & 3.88 & 5.84 & 3.69 & 0.05 \\
\hline TP-EC & 1.14 & 0.83 & 1.18 & 0.86 & 0.78 \\
\hline Decay-EC & 1.59 & 0.94 & 1.21 & 0.70 & 0.06 \\
\hline Duration EC & 2.73 & 1.30 & 2.39 & 1.22 & 0.22 \\
\hline Onset EOHUD & 7.19 & 4.22 & 7.28 & 4.20 & 0.82 \\
\hline TP-EOHUD & 1.48 & 0.92 & 1.16 & 0.95 & 0.16 \\
\hline Decay-EOHUD & 1.08 & 0.60 & 1.18 & 0.78 & 0.86 \\
\hline $\begin{array}{l}\text { Duration } \\
\text { EOHUD }\end{array}$ & 2.56 & 1.24 & 2.34 & 1.59 & 0.24 \\
\hline Onset ECHUD & 5.93 & 3.95 & 6.33 & 3.95 & 0.58 \\
\hline TP-ECHUD & 1.19 & 0.97 & 1.19 & 1.22 & 0.80 \\
\hline Decay-ECHUD & 1.36 & 1.04 & 1.00 & 0.79 & 0.11 \\
\hline $\begin{array}{l}\text { Duration } \\
\text { ECHUD }\end{array}$ & 2.56 & 1.47 & 2.18 & 1.45 & 0.24 \\
\hline \multicolumn{6}{|c|}{$\begin{array}{l}\text { S.D.: Standard Deviation, EO: eyes open, EC: eyes closed, EOHUD: eyes open with head movement, ECHUD: eyes } \\
\text { closed with head movement. } \\
\text { Onset: muscle contraction onset, TP: peak muscle activation, Decay: decay of muscle contraction, Duration: muscle } \\
\text { contraction duration. }\end{array}$} \\
\hline
\end{tabular}

Table 2: Neuromuscular timing (seconds) comparisons of Gastrocnemius during single and dual tasks variables. Results of Wilcoxon Signed Rank Non-Parametric Test performed comparing EMG variables. Significance level set at $p \leq 0.01$.

\begin{tabular}{|l|l|l|l|l|l|}
\hline N=29 & $\begin{array}{l}\text { Dual Task } \\
\text { Means }\end{array}$ & $\begin{array}{l}\text { Dual Task } \\
\text { SD }\end{array}$ & $\begin{array}{l}\text { Single Task } \\
\text { Means }\end{array}$ & $\begin{array}{l}\text { Single Task } \\
\text { SD }\end{array}$ & P Value \\
\hline Onset-EO & 7.96 & 4.60 & 7.41 & 3.97 & 0.55 \\
\hline TP-EO & 0.40 & 2.14 & 0.59 & 0.53 & 0.69 \\
\hline Decay-EO & 1.64 & 2.19 & 0.93 & 0.58 & 0.25 \\
\hline Duration EO & 2.03 & 1.81 & 1.53 & 0.74 & 0.34 \\
\hline Onset EC & 7.37 & 4.35 & 7.54 & 3.99 & 0.97 \\
\hline TP-EC & 1.07 & 0.95 & 1.24 & 0.98 & 0.39 \\
\hline Decay-EC & 0.96 & 0.73 & 1.35 & 0.96 & 0.06 \\
\hline
\end{tabular}

Table. 3 To be Cont...... 


\begin{tabular}{|c|c|c|c|c|c|}
\hline Duration EC & 2.04 & 1.18 & 2.59 & 1.41 & 0.13 \\
\hline Onset EOHUD & 8.13 & 3.61 & 6.25 & 3.66 & 0.07 \\
\hline TP-EOHUD & 1.00 & 0.64 & 0.95 & 0.81 & 0.55 \\
\hline Decay-EOHUD & 0.83 & 0.53 & 101 & 1.12 & 0.80 \\
\hline Duration EOHUD & 0.83 & 0.53 & 1.96 & 1.69 & 0.001 \\
\hline Onset ECHUD & 5.22 & 3.57 & 6.36 & 3.81 & 0.30 \\
\hline TP-ECHUD & 1.07 & 0.57 & 0.92 & 0.57 & 0.55 \\
\hline Decay-ECHUD & 1.11 & 0.84 & 1.04 & 0.65 & 0.99 \\
\hline Duration ECHUD & 2.17 & 1.08 & 1.97 & 0.97 & 0.55 \\
\hline \multicolumn{6}{|c|}{$\begin{array}{l}\text { S.D.: Standard Deviation, EO: eyes open,EC: eyes closed, EOHUD: eyes open with head movement, ECHUD: eyes } \\
\text { closed with head movement. } \\
\text { Onset: muscle contraction onset, TP: peak muscle activation, Decay: decay of muscle contraction, Duration: muscle } \\
\text { contraction duration. }\end{array}$} \\
\hline
\end{tabular}

Table 3: Neuromuscular timing (seconds) comparisons of Tibialis Anterior during single and dual tasks variables. Results of Wilcoxon Signed Rank Non-Parametric Test performed comparing EMG variables. Significance level set at $\mathrm{p} \leq 0.01$.

Table 4 depicts the corresponding neuromuscular coordination patterns between GA and TA during different single tasks. During the eyes open (EO) task, GA took longer to maximally activate (TP) compared to TA, and the duration of activity for GA was higher than TA $(\mathrm{p}=0.01)$. Table 5 illustrates similarities in coordination patterns between the two muscles during dual-tasks, though some significant distinctions were demonstrated. The average onset of TA activity (7.96+/-4.60) was higher than GA (5.24+/-4.35) during EO ( $\mathrm{p}=0.01)$. The average decay of GA contraction (1.59+/-0.94) was higher than TA $(0.96+/-0.73)$ during EC $(\mathrm{p}=0.01)$. Lastly, the average duration of GA activity $(2.56+/-1.24)$ was longer than TA $(0.83+/-0.53)$ during EOHUD $(\mathrm{p}=0.001)$.

\begin{tabular}{|l|l|l|l|l|l|}
\hline N=29 & $\begin{array}{l}\text { GA } \\
\text { Means }\end{array}$ & $\begin{array}{l}\text { GA } \\
\text { SD }\end{array}$ & $\begin{array}{l}\text { TA } \\
\text { Means }\end{array}$ & $\begin{array}{l}\text { TA } \\
\text { SD }\end{array}$ & P Value \\
\hline Onset-EO & 5.98 & 3.74 & 7.41 & 3.97 & 0.15 \\
\hline TP-EO & 1.19 & 1.31 & 0.59 & 0.53 & $\mathbf{0 . 0 1}$ \\
\hline Decay-EO & 1.36 & 1.18 & 0.93 & 0.58 & 0.22 \\
\hline Duration EO & 2.55 & 1.70 & 1.53 & 0.74 & $\mathbf{0 . 0 1}$ \\
\hline Onset EC & 5.84 & 3.69 & 7.54 & 3.99 & 0.14 \\
\hline TP-EC & 1.18 & 0.86 & 1.24 & 0.98 & 0.85 \\
\hline Decay-EC & 1.21 & 0.70 & 0.35 & 0.96 & 0.81 \\
\hline Duration EC & 2.39 & 1.22 & 2.59 & 1.41 & 0.71 \\
\hline Onset EOHUD & 7.28 & 4.20 & 6.25 & 3.66 & 0.28 \\
\hline TP-EOHUD & 1.16 & 0.95 & 0.95 & 0.81 & 0.11 \\
\hline Decay-EOHUD & 1.18 & 0.78 & 1.01 & 1.12 & 0.26 \\
\hline Duration EOHUD & 2.34 & 1.59 & 1.96 & 1.69 & 0.31 \\
\hline Onset ECHUD & 6.33 & 3.95 & 6.36 & 3.81 & 0.80 \\
\hline TP-ECHUD & 1.19 & 1.22 & 0.922 & 0.57 & 0.86 \\
\hline Decay-ECHUD & 1.00 & 0.79 & 1.04 & 0.65 & 0.25 \\
\hline Duration ECHUD & 2.18 & 1.45 & 1.97 & 0.97 & 0.80 \\
\hline $\begin{array}{l}\text { S.D.: Standard Deviation, EO: eyes open }, \text { EC: eyes closed, EOHUD: eyes open with head movement, ECHUD: eyes } \\
\text { closed with head movement. }\end{array}$ \\
$\begin{array}{l}\text { Onset: muscle contraction onset, TP: peak } \\
\text { contraction duration. }\end{array}$ & & & & \\
\hline
\end{tabular}

Table 4: Neuromuscular timing (seconds) comparisons of Tibialis Anterior and Gastrocnemius during single tasks variables. Results of Wilcoxon Signed Rank Non-Parametric Test performed comparing EMG variables. Significance level set at $\mathrm{p} \leq 0.01$. 


\begin{tabular}{|l|l|l|l|l|l|}
\hline N=29 & $\begin{array}{l}\text { GA } \\
\text { Means }\end{array}$ & $\begin{array}{l}\text { GA } \\
\text { SD }\end{array}$ & $\begin{array}{l}\text { TA } \\
\text { Means }\end{array}$ & $\begin{array}{l}\text { TA } \\
\text { SD }\end{array}$ & P Value \\
\hline Onset-EO & 5.24 & 4.35 & 7.96 & 4.60 & $\mathbf{0 . 0 1}$ \\
\hline TP-EO & 1.08 & 0.86 & 0.40 & 2.14 & 0.05 \\
\hline Decay-EO & 1.40 & 1.04 & 1.64 & 2.19 & 0.58 \\
\hline Duration EO & 2.48 & 1.59 & 2.03 & 1.81 & 0.09 \\
\hline Onset EC & 7.97 & 3.88 & 7.37 & 4.35 & 0.24 \\
\hline TP-EC & 1.14 & 0.83 & 1.07 & 0.95 & 0.83 \\
\hline Decay-EC & 1.59 & 0.94 & 0.96 & 0.73 & $\mathbf{0 . 0 1}$ \\
\hline Duration EC & 2.73 & 1.30 & 2.04 & 1.18 & 0.05 \\
\hline Onset EOHUD & 7.19 & 4.22 & 8.13 & 3.61 & 0.32 \\
\hline TP-EOHUD & 1.48 & 0.92 & 1.00 & 0.64 & 0.05 \\
\hline Decay-EOHUD & 1.08 & 0.60 & 0.83 & 0.53 & 0.13 \\
\hline Duration EOHUD & 2.56 & 1.24 & 0.83 & 0.53 & $\mathbf{0 . 0 0 1}$ \\
\hline Onset ECHUD & 5.93 & 3.95 & 5.22 & 3.57 & 0.46 \\
\hline TP-ECHUD & 1.19 & 0.97 & 1.07 & 0.57 & 0.96 \\
\hline Decay-ECHUD & 1.36 & 1.04 & 1.11 & 0.84 & 0.55 \\
\hline Duration ECHUD & 2.56 & 1.47 & 2.17 & 1.08 & 0.24 \\
\hline $\begin{array}{l}\text { S.D.: Standard Deviation, EO: eyes open, EC: eyes closed, EOHUD: eyes open with head movement, ECHUD: eyes } \\
\text { closed with head movement. }\end{array}$ \\
$\begin{array}{l}\text { Onset: muscle contraction onset, TP: peak muscle activation, Decay: decay of muscle contraction, Duration: muscle } \\
\text { contraction duration. }\end{array}$ & & & & \\
\hline
\end{tabular}

Table 5: Comparisons of Tibialis Anterior and Gastrocnemius during Dual tasks variables. Results of Wilcoxon Signed Rank Non-Parametric Test performed comparing EMG variables. Significance level set at $p \leq 0.01$.

\section{Discussion}

To our knowledge, limited studies have compared EMG patterns of lower limb musculature during single and dual-motor tasks, especially in young adult populations. This study aims to identify whether complex balance tasks evoke distinct neuromuscular patterns in TA and GA in young adults. We speculate there will be no distinguishable changes in TA and GA activity in the balance tests due to effective sensory and motor systems in young adults; although, the complexity of the tasks might elicit postural challenges. The results of our query primarily exhibit no significant changes in TA and GA EMG activity, though some changes were more prominent during dual-motor tasks. Consequently, we partially support our hypothesis.

The comparable EMG activity of the TA during single and dual-motor tasks implies effective postural control mechanisms in young adults. The similar EMG activity of GA across different balance tests indicates young adults adapt to different postural conditions by modulating sensory and motor systems. ShumwayCook and Woollacott explained how attentional resources required for preserving balance are consistent regardless if a secondary task is introduced [11]. Thence, our participants were able to distribute attentional resources to the motor task and balance tasks effectively. Nevertheless, the prolonged duration of TA activity during EOHUD could be due to the novelty of the balance task, especially when head movements are introduced in this particular task. Yet, participants continued to maintain balance without significant changes in TA activity during ECHUD, indicating adaptations to postural control.

On the contrary, the similarities in EMG activity for both muscles despite the addition of a motor task could suggest that our dualmotor tasks were elementary. Prospective studies should examine the neuromuscular activity of postural muscles during more demanding multiplex balance conditions.

Minor significant findings were identified when comparing neuromuscular patterns between TA and GA during single tasks.
GA took longer to maximally activate (TP) compared to TA during EO indicating sway was initially posterior. This outcome could be due to the unfamiliarity of the balance task; however, as previously mentioned, participants acclimated to the demands of postural stability as the complexity of the tasks increased. Additionally, the duration of the contraction of GA was greater than TA during $\mathrm{EO}$, indicating GA plays an essential role in maintaining balance persistently - a previously recognized concept $[2,22]$.

The variations in neuromuscular patterns between TA and GA during motor tasks could be due to the novelty of the motor task, resulting in delayed onset of TA activation compared to GA during the EO task. GA contraction took longer to decay compared to TA during the EC dual-motor task, which again portends the critical role GA plays in maintaining balance. We deduce canceling visual input (EO) requires sensory reweighting and alterations to postural muscle activity. To support our findings, the study by Baribieri et al. reveals increased anterior-posterior sway during EC conditions [13]. Our participants could have experienced increased sway during EC, requiring prolonged contraction of GA, which creates a plantarflexion force preventing the body from falling forward or losing balance in general. Additionally, this same inference could explain why the duration of GA contraction was longer than TA contraction during the EOHUD motor task. The inclusion of head movements along with the motor task can create dual-task interference, especially since adjustments to balance control need to be immediate. The unpredictable challenges to sensory and motor systems created by the EOHUD test demand prolonged activation of GA in order to sustain postural control. Sozzie et al. also identified alterations in EMG activity in the ankle muscles and longer time intervals than normal for modifying balance control during different balance tests [14]. This finding further emphasizes the importance of a swift interaction between sensory inputs and postural muscles which can avoid falls during stringent balance conditions. Previous studies have identified an increase in postural sway as well as EMG activity in postural musculature during 
difficult balance tasks $[10,15,16]$.

As aforementioned, several changes in EMG activity during dual-motor conditions were identified in our study, mostly in GA compared to TA. Previous literature has identified the GA as an agonist at the ankle joint, and TA as an antagonist, with the GA showing more altered EMG patterns during quiet stance similar to our study [3]. Loram and Lakie assume the soleus and gastrocnemius contribute to muscle proprioceptive information responsible for postural stability [12], however Giulio et al. proposes proprioceptive information responsible for postural stability derives from the unmodulated (no change in EMG activity) TA [3]. Thus, more studies should be conducted to understand whether TA or GA contributes more to proprioceptive input and postural stability.

The minute changes in EMG activity with the performance of a motor task was similarly found in the study by Kwon and colleagues, though this study assessed kinematic data during gait [17]. In their study, walking while holding a cup filled with water showed a significant decline in spatial-temporal parameters as well as decreased hip joint motion during the stance phase to preserve balance compared to normal gait. Changes in gait parameters were also seen when a cognitive task was introduced during gait. Similarly, to our inquiry, this study highlights the complexity of a dual-motor task and future studies should consider interpreting EMG patterns during dual-cognitive and dual-motor tasks. Another study's results suggest there is a shift in subcortical processing to cortical processing when the complexity of the balance task is increased [18]. More studies need to be conducted to determine the cortical control of postural muscles during dual-motor tasks.

The undifferentiated neuromuscular patterns for TA and GA across various balance tasks can also be explained by postural control theories. For example, the activation of synergistic postural muscles, such as hamstrings and paraspinal, ensures postural control when forces from other joints create instability [11]. Furthermore, the ideal alignment in stance requires a vertical line of gravity falling proportionate to body structures allowing for minimal energy expenditure of muscles to maintain balance. We propose that due to ideal alignment requiring the center of mass to be within the base of support, alterations in GA activity were shown in order to maintain ideal alignment, especially as the motor tasks imposed challenges to sensory processing systems. Moreover, the cutaneous sensory inputs from the soles of the feet contribute to an automatic extension force at the foot, requiring an increase in the postural tone of extensor muscles such as the GA [11]. Although our study did not explore the force output of muscles, having the participants standing on an unstable surface increases the sensory input and modifications to a motor response. Therefore, we could infer that GA force output and GA EMG activity was higher than TA due to an increase in extensor tone for balance support. Future studies should consider utilizing force plates and analyze EMG force output to comprehend how ground reaction forces play a role in changing EMG activity during balance tasks.

One limitation of our study was not having standardized foot placement on the foam pad. Some participants might have been in a wide stance allowing a larger BoS, in turn enhancing postural stability. Prospective studies should establish a standardized foot placement protocol to avoid variations in BoS. In addition, participants might have had deviations in their gaze towards the cup of water instead of the orange square to avoid water spills. Future studies should investigate whether participants change attentional gaze during dualmotor tasks incorporating head nods.

\section{Conclusion}

Our study proposes the neuromuscular activity of the TA and GA are mostly consistent regardless of the sensory conditions in young adults. However, the complex postural stability requirements involved in a dual-motor task might alter neuromuscular patterns in the lower limb musculature to compensate for any postural imbalance. Further studies should analyze the different brain structures involved in maintaining balance during dual-motor tasks as well as whether different neuromuscular patterns are identifiable during dualcognitive and dual-motor tasks. Discerning neuromuscular patterns and cognitive demands during dual-motor tasks can help clinicians find and understand methods to train fall risk patients.

Conflicts of interest/Competing interests: Authors report no conflict or competing interest.

\section{Reference}

1. Pollock, A. S., Durward, B. R., Rowe, P. J., \& Paul, J. P. (2000). What is balance? Clinical Rehabilitation. 14(4): 402-6. doi:http://dx.doi.org.ezp.twu.edu/10.1191/0269215500cr342oa

2. Sozzi S., Honeine J., Do M, Schieppati M. (2013). Leg muscle activity during tandem stance and control of body balance in the frontal plane. Clin Neurophysiol. 124(6):1175-86. doi: 10.1016/j.clinph.2012.12.001.

3. Giulio, I.D., Maganaris, C.N., Baltzopoulous, V., Loram, I.D. (2009). The proprioceptive and agonist roles of gastrocnemius, soleus and tibialis anterior muscles in maintaining human upright posture. Journal of Physiology. 587(10):2399-416. Doi: 10.1113/jphysiol.2009.168690.

4. Yoon, S-W. (2017). Analysis of the muscular activities of the tibialis anterior and gastrocnemius muscles in functional reach." Journal of Physical Therapy Science. 29(5): 851-53. doi: 10.1589/jpts.29.851

5. Shumway-Cook, A., \& Woollacott, M.H. (2007). Motor control: translating research into clinical practice. (6th ed.) Philadelphia: Lippincott Williams \& Wilkins. APA

6. D'Silva, L. J., Lin, J., Staecker, H., Whitney, S.L., Kluding, P.M. (2016). Impact of Diabetic Complications on Balance and Falls: Contribution of the Vestibular System. Physical Therapy. 96(3):400-409. doi: 10.2522/ptj.20140604.

7. Alberts, J. L., Hirsch, J. R., Koop, M. M., Schindler, D. D., Kana, D. E., Linder, S. M., Campbell, S., \& Thota, A. K. (2015). Using Accelerometer and Gyroscopic Measures to Quantify Postural Stability. Journal of athletic training, 50(6), 578-588. https://doi.org/10.4085/1062-6050-50.2.01

8. Chowdhury, R. H., Reaz, M. B., Ali, M. A., Bakar, A. A., Chellappan, K., \& Chang, T. G. (2013). Surface electromyography signal processing and classification techniques. Sensors (Basel, Switzerland), 13(9), 12431-12466. https://doi.org/10.3390/ s130912431

9. Shin, S. S., \& An, D. H. (2014). The effect of motor dualtask balance training on balance and gait of elderly women. Journal of physical therapy science, 26(3): 359-361. https://doi. org/10.1589/jpts.26.359

10. Lee, Y., Lee, J., Shin, S., Lee, S.. (2012). The effect of dual motor task training while sitting on trunk control ability and balance of patients with chronic stroke. Journal of Physical Therapy Science. 24(4): 345-49. DOI: https://doi.org/10.1589/ jpts.24.345

11. Shumway-Cook, A. and Woollacott, M.H. (2001). Motor control theory and practical applications. (2nd edition). Lippincott Williams \& Wilkins. 56-257.

12. Loram, I.D., \& Lakie, M. (2002). Direct measurement of human ankle stiffness during quiet standing: the intrinsic mechanical stiffness is insufficient for stability. J Physiol. 545(3):1041-53. doi: 10.1113/jphysiol.2002.025049.

13. Barbieri, F.A., Penedo, T., Simieli, L., Barbieri, R.A., Zagatoo, A.M., Dieen, J.H., Pijnappels, M.A.G.M., Rodrigues, S.T., Polastri, P.F. (2019). Effects of ankle muscle fatigue and visual behavior on postural sway in young adults. Front Physiol. 10, 643. 
14. Sozzie, S., Monti, A., Nunzio, A.M.D., Do, M.-C., Scieppati, M. (2011). Sensori-motor integration during stance: time adaptation of control mechanisms on adding or removing vision. Hum Mov Sci. 30(2):172-89. doi: 10.1016/j.humov.2010.06.002.

15. Cimadoro, G., Paizis, C., Alberti, G., Babault, N. (2013). Effects of different unstable supports on EMG activity and balance. Neurosci. 548:228-32. doi: 10.1016/j.neulet.2013.05.025.

16. Muehlbauer, T., Roth, R., Bopp, M., Granacher, U. (2012). An exercise sequence for progression in balance training. $\mathrm{J}$ strength Cond Res. 2:568-74. doi: 10.1519/JSC.0b013e318225f3c4.
17. Kwon, Y., Kwon, J.W., Cho, I.H. (2019). The difference gait characteristic according to the variety of dual tasks in young healthy adults. Work. 63(1): 33-38. DOI: 10.3233/WOR192905

18. Gebel, A., Lüder, B., Granacher, U. (2019). Effects of increasing balance task difficulty on postural sway and muscle activity in healthy adolescents. Front Physiol. 10:1135. doi: 10.3389/ fphys. 2019.01135 